\title{
Screening transposon-mutant library of methicillin-resistant Staphylococcus aureus for mutants with altered sensitivity toward three antimicrobial agents
}

\author{
Mahmoud Ahmed $^{1^{\star}}$, George Beshara ${ }^{1^{*}}$, Bryan Ubanwa ${ }^{2^{*}}$, Taeok Bae ${ }^{3}$ \\ ${ }^{1}$ Indiana University Northwest, Department of Biology, ${ }^{2}$ University of Houston, \\ Department of Biology, ${ }^{3}$ Indiana University School of Medicine-Northwest \\ * Equal contribution
}

Background and Hypothesis: Methicillin-resistant Staphylococcus aureus (MRSA) is resistant to almost all beta-lactam antibiotics, including oxacillin. To combat MRSA infections, recently, new classes of drugs are developed, such as oxadiazole DR1 and ESP404. We hypothesize that, if we identify MRSA mutants with altered sensitivity toward the drugs, those mutants will help us understand the antimicrobial mechanisms of the drugs.

Experimental Design or Project Methods: The screening was carried out on the Nebraska library consisting of 1,920 non-redundant transposon mutants of USA300 strain in 96 well plates. First, the minimum inhibitory concentration (MIC) of each drug was determined. Then the transposon mutants were grown in tryptic soy broth (TSB) containing either $1 / 4 \times \mathrm{MIC}$ or $4 \times \mathrm{MIC}$ of the drugs for sensitive mutants and resistant mutants respectively. Due to the limited availability of ESP404, the drug was used to screen for sensitive mutants only.

Results: The first screening identified 81 mutants with altered drug sensitivity: 23 for oxadiazole DR1 (5 sensitive, 18 resistant); 20 for ESP404 (all sensitive); 38 for oxacillin (18 sensitive, 5 resistant).

Conclusion and Potential Impact: Screening of the transposon-mutant library identified multiple mutants that have altered sensitivity toward three drugs against $S$. aureus. Further verification of the phenotype and characterization of those mutants are expected to shed light on the antimicrobial mechanism of the drugs and open a door for the development of drug-potentiators. 